\title{
Spectral Statistics of Instantaneous Normal Modes in Liquids and Random Matrices
}

\author{
Srikanth Sastry ${ }^{1}$, Nivedita Deo ${ }^{1,3}$ and Silvio Franz ${ }^{2}$ \\ 1 Jawaharlal Nehru Center for Advanced Scientific Research, Bangalore 560064, India \\ 2 The Adbus Salam International Centre for Theoretical Physics, Treieste, Italy \\ 3 Santa Fe Institute, 1399 Hyde Park Road, Santa Fe, NM 87501, USA
}

\begin{abstract}
We study the statistical properties of eigenvalues of the Hessian matrix $\mathcal{H}$ (matrix of second derivatives of the potential energy) for a classical atomic liquid, and compare these properties with predictions for random matrix models (RMM). The eigenvalue spectra (the Instantaneous Normal Mode or INM spectra) are evaluated numerically for configurations generated by molecular dynamics simulations. We find that distribution of spacings between nearest neighbor eigenvalues, $s$, obeys quite well the Wigner prediction $s \exp \left(-s^{2}\right)$, with the agreement being better for higher densities at fixed temperature. The deviations display a correlation with the number of localized eigenstates (normal modes) in the liquid; there are fewer localized states at higher densities which we quantify by calculating the participation ratios of the normal modes. We confirm this observation by calculating the spacing distribution for parts of the INM spectra with high participation ratios, obtaining greater conformity with the Wigner form. We also calculate the spectral rigidity and find a substantial dependence on the density of the liquid.
\end{abstract}

PACS numbers: 75.10.Nr, 61.20.-p, 61.43.-j, 64.70.Pf, 05.40.-a, 05.45+b 


\section{INTRODUCTION:}

The local topography of the potential energy surface, as characterized by the ensemble averaged spectrum (INM spectrum) of eigenvalues of the second-derivative matrix (Hessian) of the potential energy function, have been studied in recent years as an approach to the analysis of dynamics in liquids [1,2]. Broadly, the study of the INM spectra has been directed at analysing short time dynamics as in studying solvation [3], and at elucidating information about pathways to long time relaxation in the form of potential energy barriers etc [1,2].

Considerable effort has also been dedicated to developing analytical theories for calculating the INM spectra within an equilibrium description [4 8 . The approach in much of these attempts has been to formulate the problem of calculating the INM spectrum as an exercise in random matrix theory. If one treats the individual elements of the Hessian matrix as independent, and distributed according to the appropriate Boltzmann weight, then the Hessian may be viewed as a real, symmetric random matrix with a known distribution of matrix elements. Two properties, however, distinguish the Hessian from the standard corresponding case treated in random matrix theory: (i) The diagonal entries of the Hes-

sian are related to the off-diagonal entries by the property $\mathcal{H}_{i i}^{\alpha \beta}=-\sum_{j \neq i} \mathcal{H}_{i j}^{\alpha \beta}$, where $i, j$ label the particles and $\alpha, \beta$ the spatial coordinates $x, y, z$. (ii) For liquids with short-ranged interaction potentials, the Hessian matrix is sparse, with the fraction of non-zero entries, $p$, depending on the system size as $p \sim 1 / N$.

In view of the above considerations, it is of interest to inquire to what extent the INM spectrum displays universal features idenitied in random matrix theory. In this paper, we address this question by obtaining INM spectra numerically for a model atomic liqiud that has been studied in the context of slow dynamics in supercooled liquids [10]. The statistics we consider are the spacing statistics between nearest neighbor eigenvalues and the spectral rigidity, which we explain below. 


\section{EIGENVALUES OF THE HESSIAN}

The model liquid we study is a binary mixture [9] composed of $80 \%$ of particles of type $A$ and $20 \%$ of type $B$, interacting via the Lennard-Jones potential, with Lennard-Jones parameters $\epsilon_{A B} / \epsilon_{A A}=1.5, \epsilon_{B B} / \epsilon_{A A}=0.5, \sigma_{A B} / \sigma_{A A}=0.8$, and $\sigma_{B B} / \sigma_{A A}=0.88$, and a ratio of masses $m_{B} / m_{A}=1$. Lennard-Jones reduced units are used to report all the quantities, in terms of the $A$ particle paramters $\epsilon_{A A}, \sigma_{A A}$ and $m_{A}$ : temperatures as $T^{*}=k_{B} T / \epsilon_{A A}$, densities as $\rho^{*}=\rho / \sigma_{A A}^{3}$ and Hessian eigenvalues $\lambda^{*}=\lambda \frac{m_{A} \sigma_{A A}^{2}}{\epsilon_{A A}}$. Further details may be found in [10]. Molecular dynamics simulations of the liquid are performed at ten reduced densities $\rho^{*}$ from $\rho^{*}=0.65$ to 1.40 , at reduced temperature $T^{*}=1.0$. Two hundred sample configurations in each case are chosen from the equilibrated trajectory for the INM analysis. For each of these configurations, the Hessian is calculated and diagonalized numerically to obtain the eigenvalues $\lambda_{i}$ as well as the eigenvectors $\mathbf{e}_{i}$. The eigenvectors are used to calculate the localization properties of the normal modes, via the participation ratio

$$
P_{i}=\left[N \sum_{\alpha=1}^{3 N}\left(\mathbf{e}_{i}^{\alpha} \cdot \mathbf{e}_{i}^{\alpha}\right)^{2}\right]^{-1}
$$

The participation ratio thus defined is small (order of $1 / N$ ) for localized modes and large (order of 1) for extended modes.

By constructing the histogram of eigenvalues $\lambda$ of the Hessian for all configurations considered, we obtain the INM Density of States (DOS) or spectrum [11]. Figure 1 shows the INM spectrum $D(\lambda)$ verses $\lambda$ for different densities. Note that the DOS is very different from the Wigner semi-circle distribution, obtained in RMM. We know from the literature [13] that the correlation and spacing functions are universal in certain regions no matter what the DOS is. Thus we use the corresponding statistics of spacings between eigenvalues, Wigner-Dyson statistics, as the standard of reference. 


\section{UNFOLDING THE SPECTRUM}

The statistical analysis of the numerical data proceeds by first using an unfolding procedure. The numerical calculation yields the eigenvalues of the Hessian which is ordered and forms the sample spectrum $\left\{\lambda_{1}, \lambda_{2}, \ldots, \lambda_{n}\right\}$. In order to analyse the spacing statistics, one must transform the eigenvalues $\lambda_{i}$ in such a way that the transformed eigenvalues $\zeta_{i}$ are uniformly distributed. That is, the spectral density $D(\zeta)=1$. This procedure is referred to as "unfolding" the spectrum 14,12]. In general such a transformation for a spectral density function $D(\lambda)$ is most easily accomplished through its cumulative distribution

$$
C(\lambda) \equiv \int_{-\infty}^{\lambda} D(\lambda) d \lambda
$$

by defining $\zeta(\lambda)=C(\lambda)$. For a discrete spectrum such as the ones we consider here, the corresponding procedure would be to extract from the sample spectrum the "smooth" part of the "staircase" cumulative distribution [12]. In this work, the procedure we adopt to estimate the smooth part of the cumulative distribution is to evaluate the cumulative distribution for the union of all eigenvalues $\lambda_{i}$ obtained for 200 independent configurations at each density and temperature value. Such a smooth cumulative spectrum along with the "staircase" cumulative spectrum for a single configuration, is shown in Fig. 2, and the unfolding procedure is indicated.

\section{SPACING DISTRIBUTION}

The spacing distribution $P(s)$ for the random matrix models is defined as the probability of finding the next nearest neighbor eigenvalue of the spectrum to be at a distance s i.e. $s_{i}=\frac{\lambda_{i+1}-\lambda_{i}}{\Delta}$ where $\Delta$ is the mean level spacing. In the present case where we use unfolded eigenvalues, $s_{i}=\zeta_{i+1}-\zeta_{i}$. Then $P(s)=A s^{\beta} e^{-B s^{2}}$ where $\beta=1$ for the orthogonal random

matrix model, which is the "Wigner surmise" [14]. This spacing distribution arises in various quantum systems which show an underlying classically chaotic behavior e.g. quantum billiards, quantum dots, nuclear spectra, disordered mesoscopic systems. The system we 
analyze, on the other hand, is a classical liquid, with a disordered microscopic structure. The spacing distributions obtained are shown in Fig. 3 for three densities of the liquid. We find that to a first approximation, the cases considered display the universal behavior according to the Wigner surmise, with the agreement being better for the liquid at higher densities. We note here that in Ref. [8] a sparse random matrix is used to calculate the INM DOS analytically for a one-dimensional system which is very different from the usual Wigner semi-circle, and displays qualitative features very similar to the DOS we calculate numerically here. It would be very interesting to see whether the correlators of the sparse random matrix proposed in ref. [8] captures the above behaviour for different densities for the liquid.

\section{SPECTRAL RIGIDITY}

We next study the spectral rigidity, which measures the fluctuations of the number of eigenvalues in a window of given size as a function of the size of the window (or equivalently, the average number of eigenvalues expected in the window). The number fluctuations are plotted as a function of the mean number of eigenvalues in Fig. 4 for the same three densities as in the preceding figures. For the case of the Poisson spectrum and the harmonic oscillator [14] the number fluctuations are $\left\langle\delta N^{2}>\sim<N>\right.$ and $\left\langle\delta N^{2}\right\rangle=$ constant, while for the Gaussian random matrix ensembles $\left\langle\delta N^{2}\right\rangle \sim \ln \langle N\rangle$ for $N \gg 1$.

The number fluctuations are of the form $<\delta N^{2}>\sim N^{\gamma}$ where $\gamma(\rho)$ depends on the liquid's density. This situation is reminescent of that found for the mobility edge in the quantum hall effect and in the Anderson metal-insulator transition. A detailed analysis of this awaits future work.

\section{PARTICIPATION RATIO}

In order to get some insight into the reason for the systematically better agreement with increasing density of the spacing distribution, we consider the localization properties 
of the normal modes in the liquid. In the standard random matrix case for orthogonal matrices, the eigenvectors are all extended, while from previous numerical and analytical studies we know that a fraction of the INM eigenmodes are localized. As described earlier, we use the participation ratio to quantify the localization of modes, averaging over modes corresponding to eigenvalues in each histogram bin, for the unfolded eigenvalues. In Fig. 5, the participation ratio is plotted as a function of the unfolded eigenvalues for $T^{*}=1.00$, for values of the density $\rho^{*}=0.65,1.00$ and 1.35 . One notes that for the highest density $\rho=1.35$ the participation ratios are highest overall, while for the lower densities the participation ratios are quite small for a substantial fraction of the eigenmodes, indicating a large number of localized modes.

Next, we calculate the spacing distribution for $\rho^{*}=1.35$ for unfolded eigenvalues between 0.1 and 0.6 for which the participation ratio is high (>0.75) and relatively unchanging (Fig. 50. The resulting spacing distribution is shown in Fig. 6, along with the spacing distribution for the entire eigenvalue spectrum and the expectation based on the RMM result. The data shown clearly demonstrate that the spacing distribution is practically identical to the standard RMM result, confirming the speculation that the increasing fraction of localized states at lower densities are responsible for the deviations at these densities from the standard RMM result. Indeed, this observation has been used, in a different context to locate the mobility edge in disordered systems 20

\section{CONCLUSIONS}

We have presented the spacing statistics and spectral rigidity for numerically calculated INM spectra. The spacing statistics is seen to conform better with increasing density (at fixed temerature), with the predictions for random matrix models. We demonstrate that the source of deviations from RMM predictions is related to the presence of localized instantaneous normal modes in the liquid, whose number is greater for lower density. As the

two features distinguishing the INM spectra from the standard random matrix case are the 
nature of the diagonal elements and the sparseness of the Hessian matrix, further understanding of the non-universality of the INM spectra are to be sought in the manner in which these aspects affect the INM spectral statistics.

Acknowledgements:

We would like to thank S. Jain, V. E. Kravtsov, A. Cavagna, I. Giardina, P. J. Garrahan and P. Carpena for very useful discussions during the course of this work. SF thanks the Jawaharlal Nehru Center for Advanced Scientific Research for hospitality. 


\section{FIGURES}

FIG. 1. INM Density of States shown for three densities. Inset shows the DOS on a logarithmic scale. Eigenvalues $\lambda$ are expressed in units of $\frac{\epsilon_{A A}}{m_{A} \sigma_{A A}^{2}}$, and $D(\lambda)$ in units of $\frac{m_{A} \sigma_{A A}^{2}}{\epsilon_{A A}}$.

FIG. 2. A portion of the staircase cumulative spectrum from a single configuration is shown, along with the estimate of the smooth part of the cumulative distribution, obtained from combining eigenvalues from 200 configurations, for density $\rho^{*}=1.0$ and temperature $T^{*}=1.0$. The arrows indicate the mapping of any given eigenvalue $\lambda_{i}$ to the unfolded eigenvalue $\zeta_{i}$. The inset shows the cumulative spectra for the full range. Eigenvalues $\lambda$ are expressed in units of $\frac{\epsilon_{A A}}{m_{A} \sigma_{A A}^{2}}$, and cumulative probabilities $\zeta$ are dimensionless.

FIG. 3. The level spacing distribution $P(s)$ for densities $\rho=0.65,1.0,1.35$. Level spacings $s$ are expressed in units of $\frac{\epsilon_{A A}}{m_{A} \sigma_{A A}^{2}}$, and $P(s)$ in units of $\frac{m_{A} \sigma_{A A}^{2}}{\epsilon_{A A}}$.

FIG. 4. Spectral rigidity: Lines are fits to the form $\left\langle N^{2}>-<N>^{2} \sim<N>^{\gamma}\right.$, and the values of $\gamma$ are $1.036,0.85,0.765$ respectively for $\rho^{*}=0.65,1.00,1.35$.

FIG. 5. Participation ratio as a function of unfolded eigenvalues $\zeta$ (see caption of Fig. 2).

FIG. 6. The level spacing distribution shown for $\rho^{*}=1.35$ for the full eigenvalue spectrum (open rhombs), for the range of eigenvalues with high participation ratio ( 0.75) (filled rhombs),

along with the RMM prediction. Level spacings $s$ are expressed in units of $\frac{\epsilon_{A A}}{m_{A} \sigma_{A A}^{2}}$, and $P(s)$ in units of $\frac{m_{A} \sigma_{A A}^{2}}{\epsilon_{A A}}$. 


\section{REFERENCES}

Email: ${ }^{1}$ sastry@jncasr.ac.in, ${ }^{2}$ ndeo@jncasr.ac.in, ${ }^{3}$ franz@ictp.trieste.it.

[1] T. Keyes, J. Chem. Phys. A 101, 2921, (1997) and references therein.

[2] F. Sciortino and P. Tartaglia, Phys. Rev. Lett. 78, 2385 (1997).

[3] R. M. Stratt and M. Cho, J. Chem. Phys. 100, 6700, (1994).

[4] B.-C. Xu and R. M. Stratt, J. Chem. Phys. 92, 1923 (1990).

[5] T. M. Wu and R. F. Loring, J. Chem. Phys. 97, 8568, (1992).

[6] Y. Wan and R. M. Stratt, J. Chem. Phys. 100, 5123, (1994).

[7] G. Biroli and R. Monasson, J. Phys. A: Math. Gen. 32, L255 (1999).

[8] A. Cavagna, I. Giardina and G. Parisi, Phys. Rev. Lett. 83, 108 (1999).

[9] W. Kob and H. C. Andersen, Phys. Rev. E 51, 4626 (1995); K. Vollmayr, W. Kob and K. Binder J. Chem. Phys. 105, 4714 (1996).

[10] S. Sastry, P. G. Debenedetti and F. H. Stillinger, Nature 393, 554 (1998).

[11] Note that we have not followed the standard practing in the INM literature of plotting the DOS against $\omega \equiv \operatorname{sign}(\lambda) \times \sqrt{|\lambda|}$.

[12] T. Guhr, A. Muller-Groeling and H. A. Weidenmuller, Physics Reports, 299, Number 4-6, (1998) and references therein.

[13] E. Brézin and A. Zee, Nucl. Phys. B 402, 613 (1993), Phys. Rev. E 49, 2588 (1994).

[14] M. L. Mehta (1991) Random Matrices, Academic Press, San Diego.

[15] P. Cizeau and J. P. Bouchaud, Phys. Rev. E 50, 1810, (1994).

[16] A. J. Bray and G. J. Rodgers, Phys. Rev. B 38, 11461, (1988); G. J. Rodgers and A. J. Bray, Phys. Rev. B 37, 3557 (1988). 
[17] G. J. Rodgers and C. De Dominicis, J. Phys. A Math. Gen. 23, 1567 (1990).

[18] A. D. Mirlin and Y. V. Fyodorov, J. Phys. A: Math. Gen. 24,2273 (1991); Y. V. Fyodorov and A. D. Mirlin, Phys. Rev. Lett. 67, 2049 (1991).

[19] M. Mezard, G. Parisi and A. Zee, http://arXiv.org/abs/cond-mat/9906135.

[20] P. Carpena and P. Bernaola-Galvań Phys. Rev. B 60, 201 (1999). 


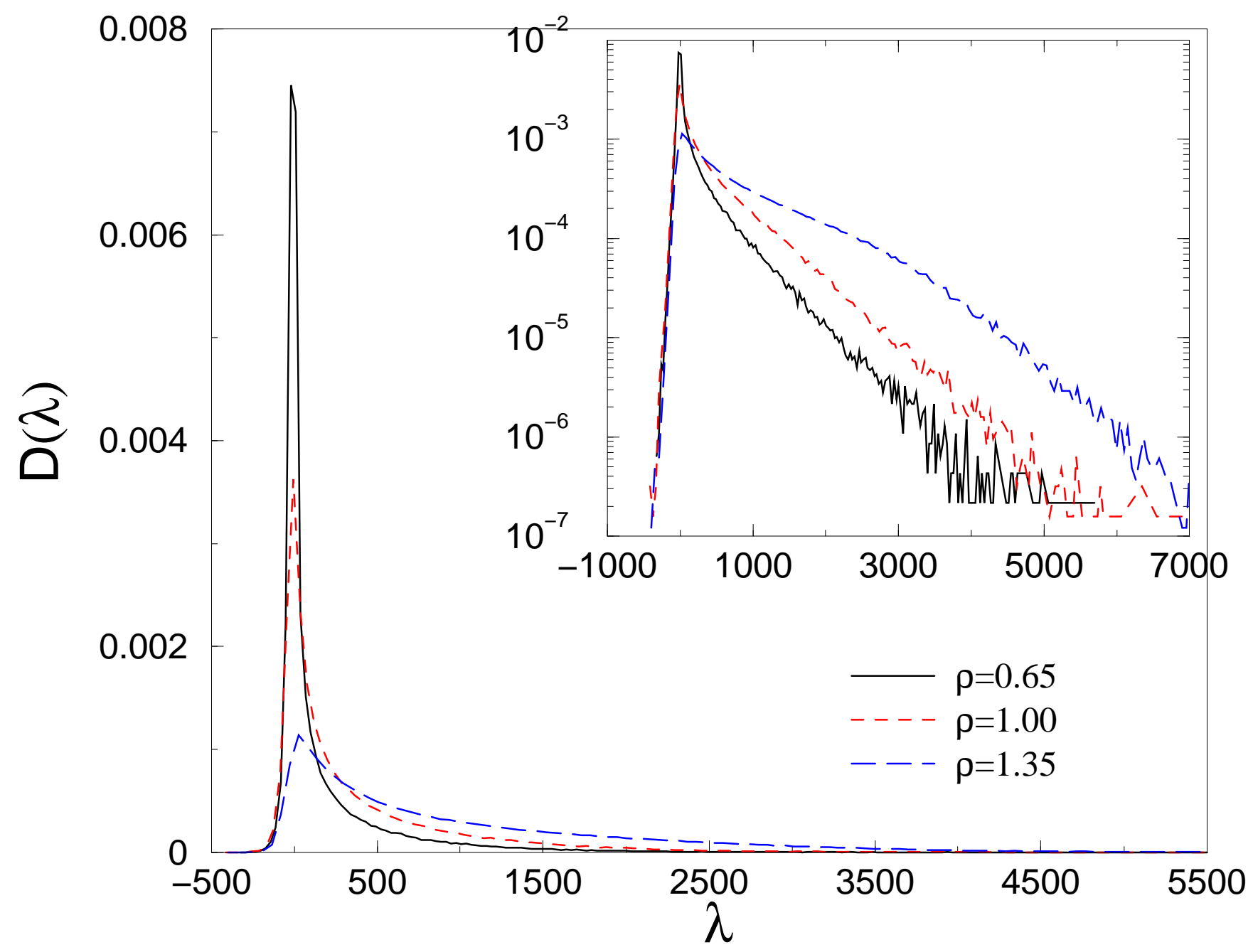




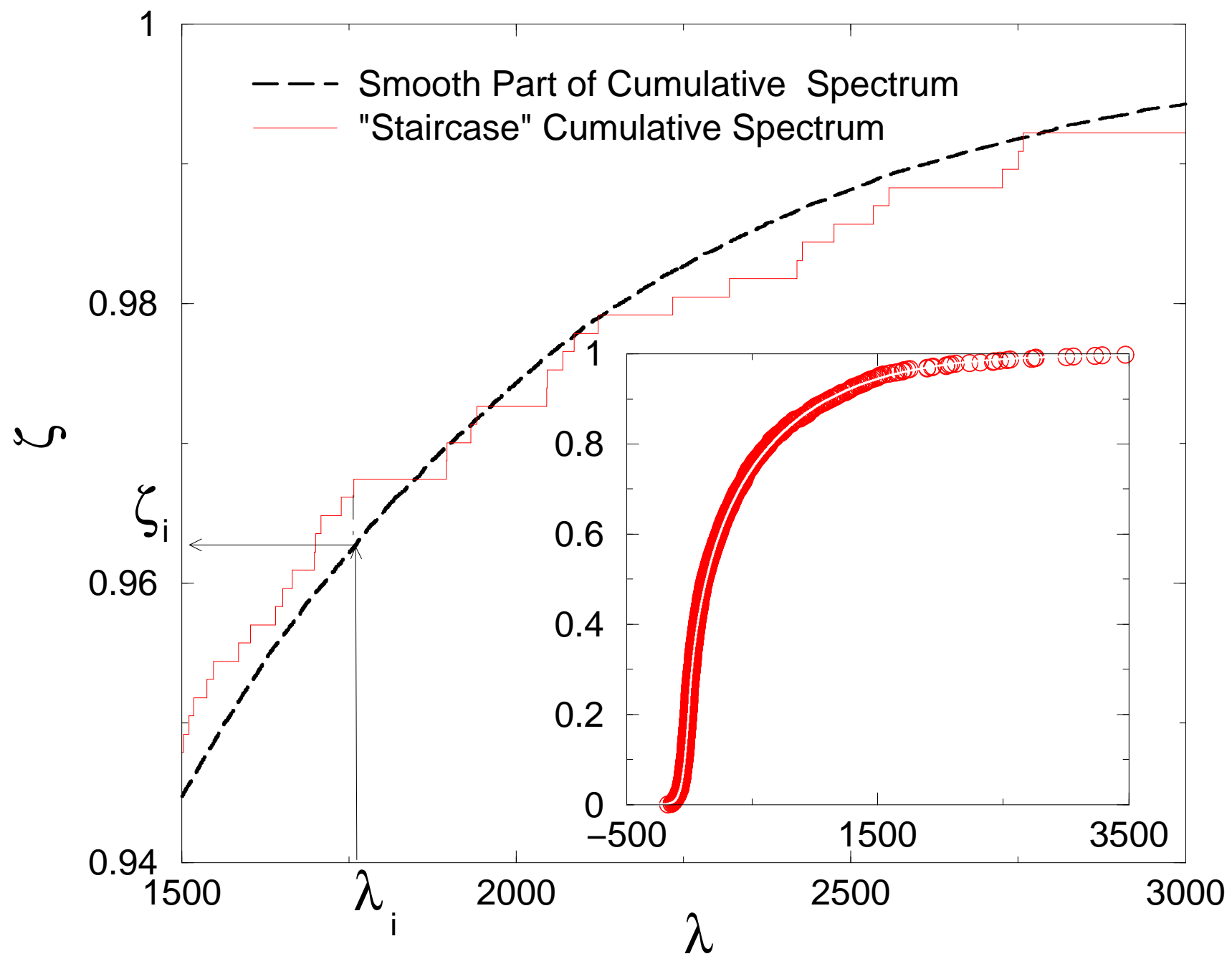




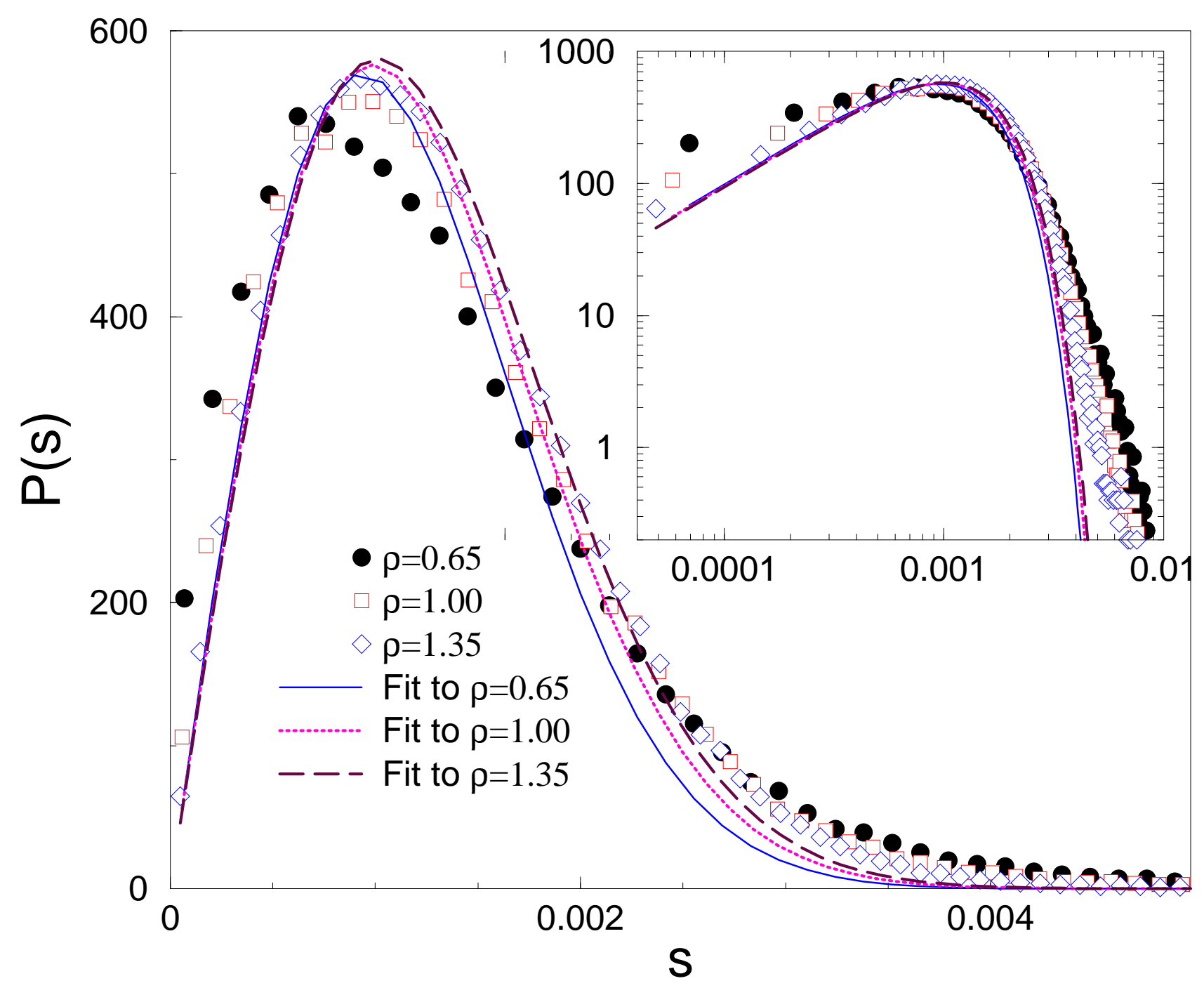




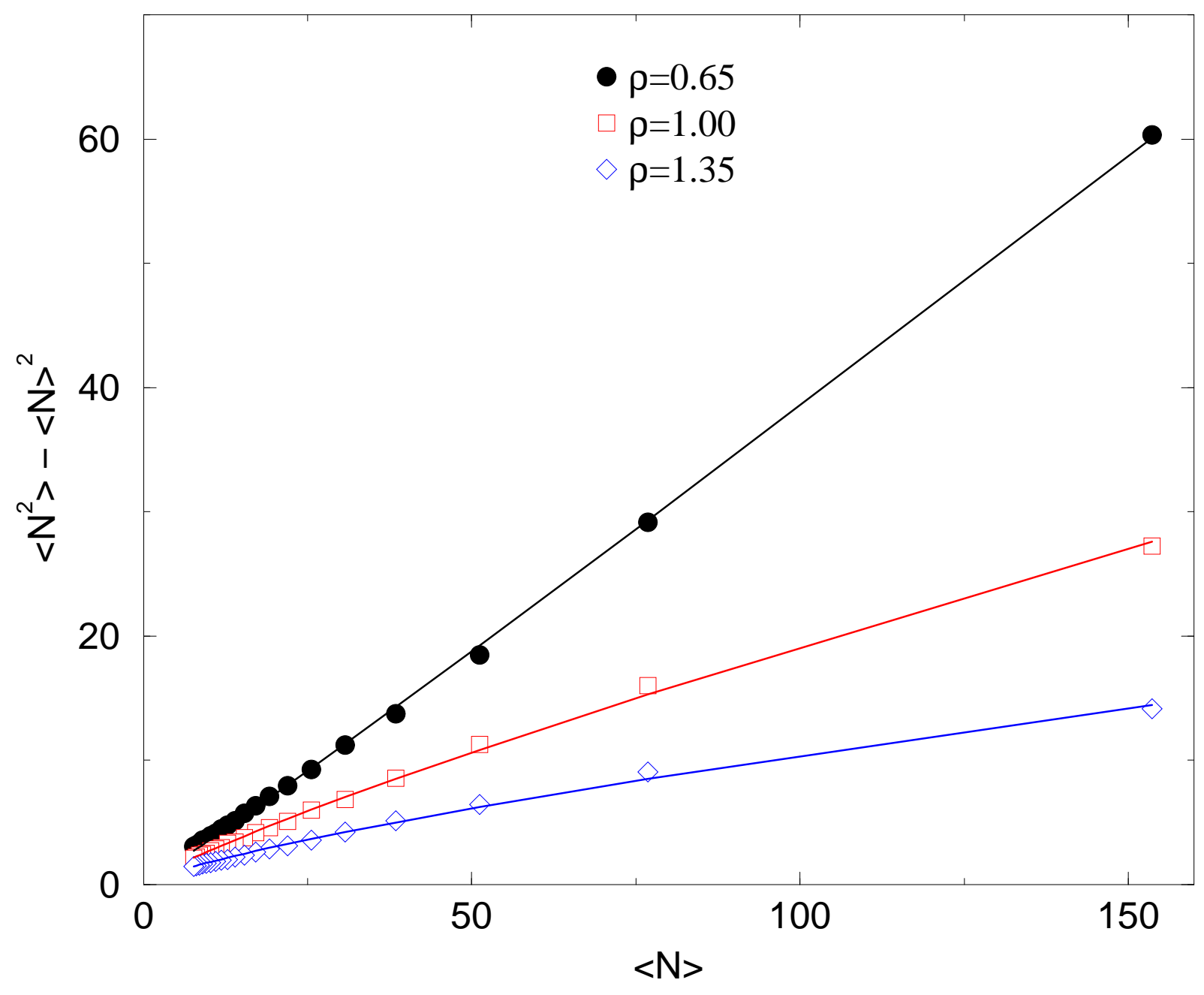




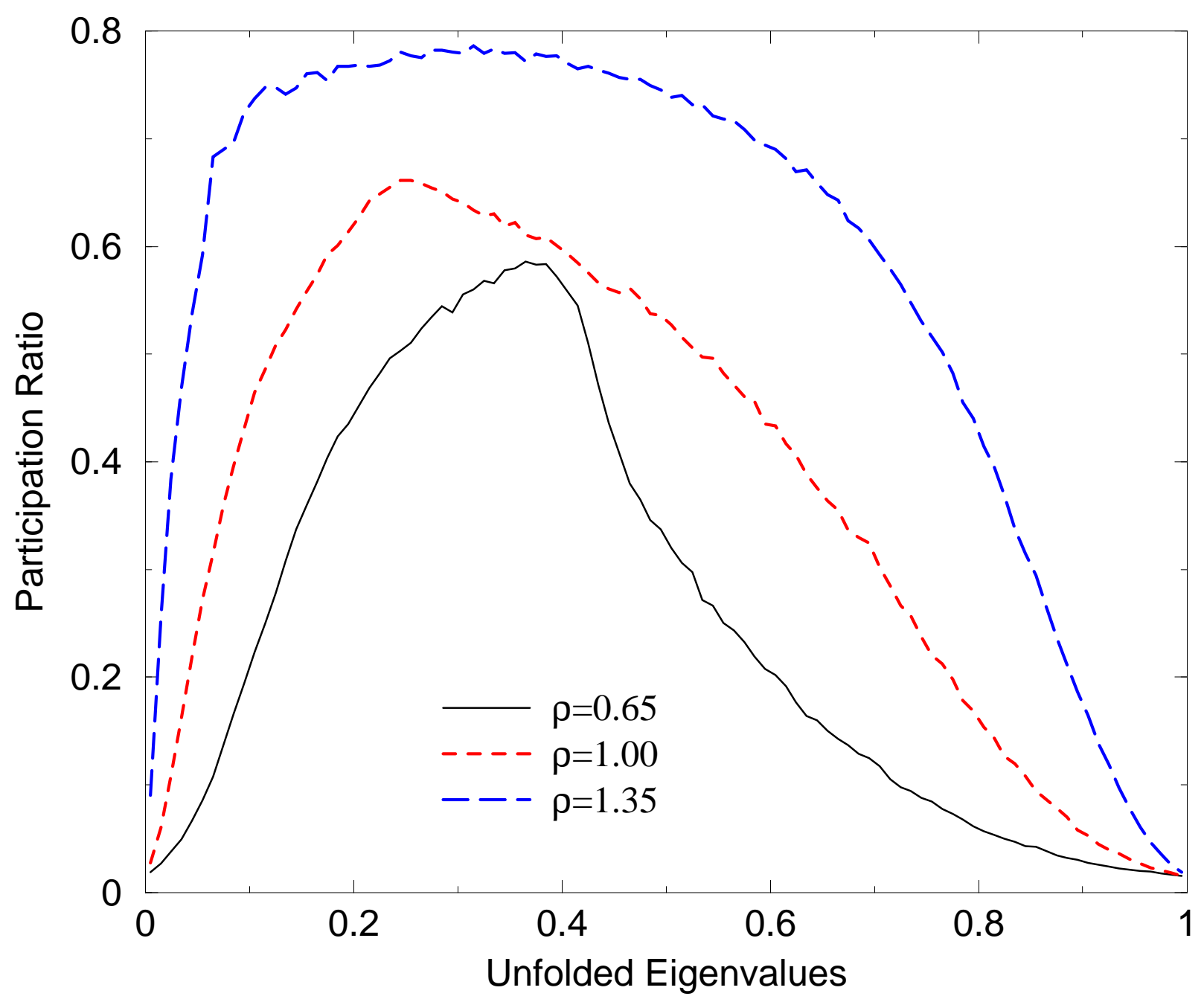




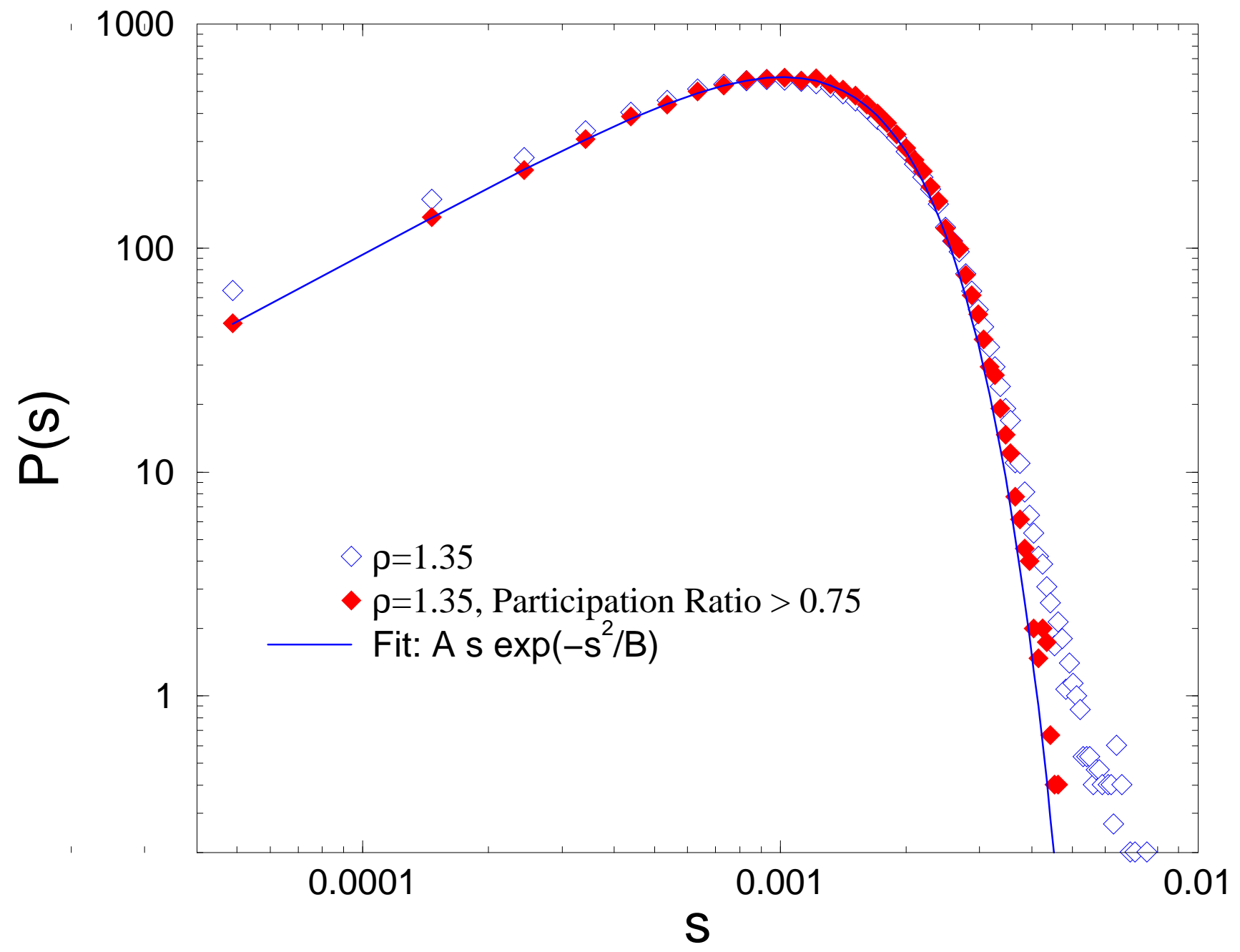

